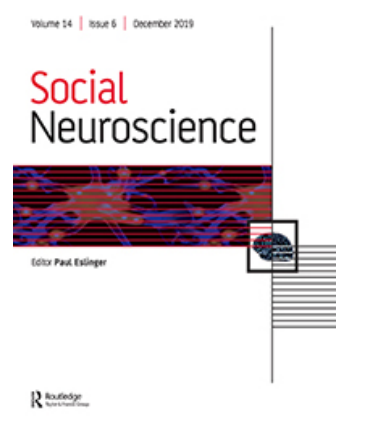

Social Neuroscience

\title{
Brain circuits involved in understanding our own and other's internal states in the context of romantic relationships
}

Sofia Esménio, José Miguel Soares, P. Oliveira-Silva, Óscar F. Gonçalves, Jean Decety \& Joana Coutinho

To cite this article: Sofia Esménio, José Miguel Soares, P. Oliveira-Silva, Óscar F. Gonçalves, Jean Decety \& Joana Coutinho (2019) Brain circuits involved in understanding our own and other's internal states in the context of romantic relationships, Social Neuroscience, 14:6, 729-738, DOI: 10.1080/17470919.2019.1586758

To link to this article: https://doi.org/10.1080/17470919.2019.1586758

Accepted author version posted online: 26

Feb 2019.

Published online: 08 Mar 2019.

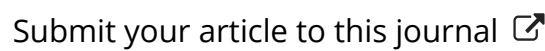

Џll Article views: 232

Q View related articles $\sqsubset$

View Crossmark data \lceil 


\title{
Brain circuits involved in understanding our own and other's internal states in the context of romantic relationships
}

\author{
Sofia Esménio $\mathbb{1}^{\mathrm{a}}$, José Miguel Soares ${ }^{\mathrm{b}, \mathrm{c}}, \mathrm{P}$. Oliveira-Silva ${ }^{\mathrm{d}}$, Óscar F. Gonçalves ${ }^{\mathrm{a}, \mathrm{e}}$, Jean Decety ${ }^{\mathrm{f}}$ \\ and Joana Coutinho \\ aPsychological Neuroscience Laboratory, Psychology School, Minho University, Braga, Portugal; bife and Health Sciences Research Institute \\ (ICVS), School of Health Sciences, University of Minho, Braga, Portugal; ‘CVS/3B's - PT Government Associate Laboratory, Braga/Guimarães, \\ Portugal, Clinical Academic Center, Braga, Portugal; 'Faculty of Education and Psychology, Catholic University of Portugal, Porto, Portugal; \\ eSpaulding Center for Neuromodulation, Spaulding Rehabilitation Hospital, Harvard Medical School, Boston, MA, USA; ${ }^{\text {fDepartment of }}$ \\ Psychology, University of Chicago, Chicago, IL, USA
}

\begin{abstract}
Social interactions require the capacity to understand both our and other's internal states. These semi-independent skills, the ability to understand oneself and others, seem to rely on the same type of representations and recruit similar brain areas. In this study, we looked at the neural basis of self and other processing in the context of an interaction with a significant other. Fourty-two participants in a monogamous relationship completed an fMRI task in which they watched a set of videovignettes of his/her romantic partner expressing emotional contents. Participants were asked to elaborate on his/her spouse's experience (other condition) and on his/her own experience when watching the video-vignettes (self-condition). The results showed a significant overlap in the brain activation for both conditions (e.g. anterior insula, posterior cingulate/precuneus, inferior frontal gyrus, inferior parietal lobule). In addition, the self-condition recruited brain areas associated with interoceptive processing and affect sharing (e.g., posterior insula), whereas the other-condition engaged brain areas involved in the cognitive representation of another's internal states and selfother distinction (e.g., fusiform, supramarginal gyrus, angular gyrus and temporoparietal junction).
\end{abstract}

ARTICLE HISTORY

Received 18 September 2018 Revised 23 January 2019 Published online 07 March 2019

\section{KEYWORDS}

Social cognition; theory of mind; self/other processing; romantic relationships

\section{Introduction}

The ability to make sense of what we ourselves and others feel or think is at the center of our capacity to interact with others. There is a general consensus, among social neuroscience researchers, that this capacity to understand internal states, either ours or those of others, recruits two main pathways: a socio- affective route (de Vignemont \& Singer, 2006), and a socio-cognitive route (Frith \& Frith, 2005). The socio-affective pathway corresponds to embodied, visceral or bottom up dimensions of social processing (Decety \& Lamm, 2006; Singer \& Lamm, 2009), while the socio-cognitive pathway refers to conceptual or top down processes that involves higher cognitive functions (Carrington \& Bailey, 2009; Frith \& Frith, 2005).

Research shows that these two pathways engage independent brain networks (Kanske, Böckler, Trautwein, \& Singer, 2015). The socio-affective route recruits areas such as the anterior cingulate cortex (ACC), anterior midcingulate cortex (aMCC) extending into the supplementary motor area (SMA), anterior insula (Al), inferior frontal gyrus (IFG), posterior cingulate (PCC), anterior thalamus, the middle temporal gyrus (MTG),midbrain and inferior parietal lobe (IPL) -including the supramarginal gyrus (SMG) and angular gyrus. (Bzdok et al., 2012; Fan, Duncan, de Greck, \& Northoff, 2011). On the other hand, the sociocognitive pathway which are often termed as Theory of Mind (TOM), or mentalizing recruits a network of brain regions that includes the posterior/ventral temporoparietal junction (TPJ), precuneus, MTG, superior temporal sulcus (STS), temporal poles and medial prefrontal cortex (mPFC) (Bzdok et al., 2012; Schurz, Radua, Aichhorn, Richlan, \& Perner, 2014).

Although both socio-affective and socio-cognitive pathways have been extensively investigated, research has only recently start looking at how these two pathways interact when processing our own and other's internal states in situations in real social life (Lamm, Decety, \& Singer, 2010; Zaki, Weber, Bolger, \& Ochsner, 2009). In fact, the majority of the experimental studies in social cognition use paradigms with fictional targets (e.g., Singer et al., 2006) or relatively artificial situations, with very few studies looking at the brain's response in real

CONTACT Sofia Esménio sof.esm@gmail.com E Psychological Neuroscience Laboratory, Psychology School, Minho University, Campus de Gualtar, 4710-057 Braga

() 2019 Informa UK Limited, trading as Taylor \& Francis Group 
interpersonal interactions. This is important because in naturally occurring social encounters there are several factors that influence the neural response to other's emotions such as the affective proximity with the target, familiarity (Cialdini, Brown, Lewis, Luce, \& Neuberg, 1997), and how much protection or care (Batson, Lishner, Cook, \& Sawyer, 2005) the target needs. Another aspect that characterizes our most significant interpersonal interactions such as parent-child or couples' interaction, is that we often have to empathize with emotional contents that are directed towards us and thus often have to alternate between the focus on our internal states and those of the other

Research in social neuroscience suggests that the understanding of other's feelings and mental states rely on the same type of representations necessary to understand our own internal states (Lamm, Bukowski, \& Silani, 2016). Accordingly, previous studies have found that similar brain areas are recruited when processing self or other internal states in both affective (e.g. empathy for pain) (Lamm et al., 2010; Singer et al., 2004), as well as cognitive experimental paradigms (e.g. reasoning about oneself/mentalizing) (Lombardo et al., 2010; Mitchell, Macrae, \& Banaji, 2006). In agreement with these findings, Dimaggio, Lysaker, Carcione, Nicolò, and Semerari (2008) suggested that the ability to understand oneself and others are "semi-independent skills". Indeed, recent evidence has also found that enhancing the ability to understand our own states increases the ability to understand those of another (Böckler, Herrmann, Trautwein, Holmes, \& Singer, 2017). This may be particularly relevant for the context of romantic relationships, if we consider evidence from simulation theories of social cognition suggesting that the closer the other is to oneself, the more likely we are to base inferences about them on the knowledge about oneself (Adolphs, 2002; Gallese \& Goldman, 1998).

However, as mentioned before, so far, the attempts to investigate the interdependence of self and other processing in the context of a meaningful relationship with a significant other, in particular in romantic relationships, are still scarce. Hence, the present study investigates the brain mechanisms involved in our ability to understand oneself and a romantic partner.

Indeed, romantic relationships are the central relationship for most adults, having an important duration in human's life cycle (Robles \& Kiecolt-Glaser, 2003). They constitute a rich source of emotional interchange (Gottman \& Levenson, 1988), highly dependent on feelings of understanding, support and empathic validation (Péloquin \& Lafontaine, 2010; Waldinger, Hauser, Schulz, Allen, \& Crowell, 2004). In addition, our choice for focusing on romantic relationships was justified by the well- documented negative impact of marital conflict on the physical and mental health of each partner (e.g., Beach, Fincham, \& Katz, 1998; Coyne et al., 2001; Greene \& Griffin, 1998), namely with important alterations in cardiovascular, endocrine and immune function (Mayne, O'leary, McCrady, Contrada, \& Labouvie, 1997). Finally, marital conflict has important social consequences not only for those directly involved in the conflict, but also for other family members, particularly children (Cummings \& Davies, 2002).

In order to address our research question we built an ecological functional magnetic resonance imaging (fMRI) task in which participants were instructed to watch a set of emotional video vignettes of their respective partner and was asked to elaborate on his/ her partner's experience (other condition) or on his/her own experience (self-condition).

Therefore, the main aim of this study is to determine the common and specific brain pathways involved in both the processing of the individual's own internal states as well as in the processing of a significant other's internal states. Regarding the common pathways, we predict a significant overlap between the self and other brain activations. This overlap should include socio-affective areas, such as ACC, aMCC, SMA, Al, PCC, anterior thalamus, IFG, IPL and midbrain (Bzdok et al., 2012; Fan et al., 2011), as well as socio- cognitive areas such as the TPJ, precuneus, STS, temporal poles and mPFC (Bzdok et al., 2012; Schurz et al., 2014). On the other hand, the capacity to understand one's internal states and to understand those of another should not be reducible to one another. Accordingly, we hypothesize that the self condition will also recruit brain areas associated with sensory and interoceptive processing, such as the posterior insula, somatosensory cortex and the SMA (Chang, Yarkoni, Khaw, \& Sanfey, 2012; Craig, 2003; Khalsa et al., 2017; Singer et al., 2004); while the other condition should engage to a further extent brain areas involved in mentalizing/TOM, including areas of perceptual processing, such as the inferior occipital gyrus, fusiform gyrus, superior temporal gyrus (Adolphs, 2002), and regions responsible for self-other distinction, namely the rTPJ and rSMG (Lamm et al., 2016; Steinbeis, 2016).

\section{Method}

\section{Participants}

Forty-two participants $(\mathrm{N}=42)$ in a committed monogamous romantic relationship for at least one year participated in this study. Prior to any procedure, all participants were screened on the telephone to assess inclusion and exclusion criteria. The exclusion criteria included: (1) the presence of any dementia and/or 
diagnosed neuropsychiatric and/or neurodegenerative disorder; (2) dependency or abuse of alcohol and/or drugs in the past year; (3) inability to attend the MRI session (e.g., metallic implants; pregnancy); (4) age below 20 or above 50 years.

All the participants were Caucasian, right-handed and the age of the participants ranged from 23 to 40 years old $(M=31.17, S D=4.748$; for men: $M=32.13, S D=4.893$, for women: $M=30.22$, $S D=4.502)$. Regarding the characteristics of the relationship: (1) $30,4 \%$ of the couples were married; (2) $39,1 \%$ were living together; (3) $30,4 \%$ were in dating relationships; (4) $34.8 \%$ of the couples had children. In the final sample the duration of the relationships ranged from 1 to 15 years $(M=7.78 ; S D=4.17)$. Before the implementation of the study all the goals and procedures were explained to the participants that provided an informed written consent.

All the procedures were approved by the University Institutional Review Board and complied with the principles expressed in the Declaration of Helsinki (with the amendment of Tokyo 1975, Venice1983, HongKong 1989, Somerset West1996, Edinburgh 2000).

\section{Experimental task}

In this task each participant watched a series of videosvignettes of his/her romantic partner expressing personal emotional contents, and was asked to focus on his/her spouse's experience (other condition) or on his/her own experience (self condition) while listening to the content expressed in the video. The video-vignettes used in this task were extracted from a previously recorded real interaction task performed in the lab, where the couple communicated positive and problematic topics in their relationship. In this interaction task, participants were asked to disclose, one at a time, things that they would either like or dislike concerning their partner. The negative and positive aspects were selected by the spouses from a list of possible topics that was provided to them before the interaction. Participants could choose other relevant topics to discuss. Examples of negative topics included things that made them feel upset or bothered, things that they would like to change or things they didn't like about their partner. Examples of positive topics included things that made them feel happy, things they truly admire about their partner, things that they would like to keep as it is.

This interaction was videotaped and a team of independent coders segmented the entire videotaped interaction task and selected the most salient 20-second negative and positive excerpts of the video. These negative and positive video vignettes were then used to construct a functional magnetic resonance imaging (fMRI) paradigm. Neutral videos were extracted from the (EMDB) Emotional Movie Database (Carvalho, Leite, Galdo-Álvarez, \& Gonçalves, 2012). The specifics of this interaction task are described in further detail in Coutinho et al. (2017) and Coutinho et al. (2018).

The task consisted of two blocks (self or other condition), where each block contained 22 trials comprising the three different conditions: positive communications (8 trials), negative communications (8 trials) and neutral videos (6 trials). Each trial consisted of: (1) fixation cross (5 seconds); (2) instructions in accordance with the present block (e.g. "In the next movie focus on how your partner is feeling.") (3 seconds); (3) video vignette (20 seconds); (4) behavioural response (4 seconds). An example of an emotional and a neutral trial is displayed in Figure 1.

Regarding the behavioural response, participants were required to choose among one of three possible responses dependent on the emotional impact of the vignette: (1) "Bad" for any kind of discomfort, negative state or emotion - e.g. distress, feeling rejection, incomprehension, abandonment; (2) "Neutral" in the absence of any positive or negative state or emotion; or (3) "Good" for any kind of positive state or emotion - e.g. happiness, ease, acceptance, love, connection or comprehension.

Stimuli were displayed in a pseudo-randomized order that remained the same across blocks. Finally, blocks were displayed in a randomized order across participants. The total duration of the task was $1364 \mathrm{~s}$ (24min).

\section{Neuroimage acquisition}

Structural and functional images were acquired with a clinical approved 3T MRI scanner (Siemens Magnetom Tim Trio, Erlangen, German). The imaging session comprised one structural scan (192 sagittal slices, repetition

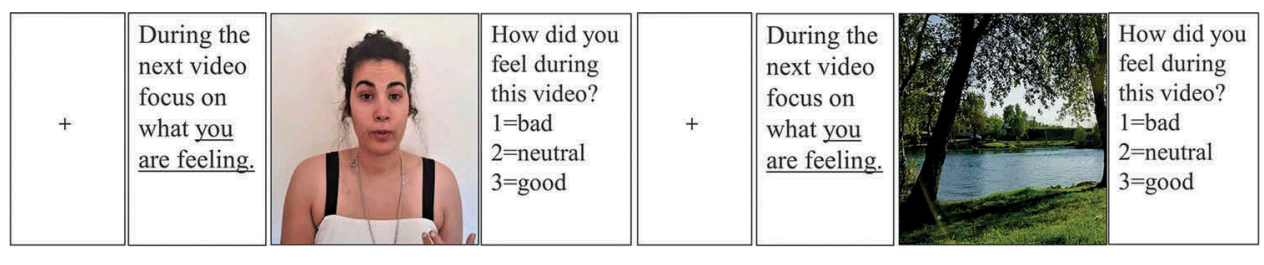

Figure 1. Scheme of an emotional and a neutral trial in the self condition. 
time $(T R)=2000 \mathrm{~ms}$; echo time $(T E)=2.33 \mathrm{~s}$, flip angle $=7^{\circ}$, slice thickness $=0.8 \mathrm{~mm}$, slice gap $=0 \mathrm{~mm}$, pixel size $=0.8 \times 0.8 \mathrm{~mm}^{2}$, field of view $\left.(\mathrm{FoV})=256 \mathrm{~mm}\right)$ and one functional blood oxygen level dependent (BOLD) sensitive echo-planar imaging (EPI) sequence (39 axial slices; repetition time $(T R)=2000 \mathrm{~ms}$; echo time $(\mathrm{TE})=29 \mathrm{~ms}$, flip angle $(\mathrm{FA})=90^{\circ}$, matrix size $=64 \times 64$, slice thickness $=3 \mathrm{~mm}$, pixel size $=3 \times 3 \mathrm{~mm}^{2}$, field of view $($ FoV $)=222 \times 222 \mathrm{~mm}$ ) were collected for each participant. The synchronization between the paradigm and the acquisition were guaranteed for each TR.

\section{Data preprocessing and analysis}

The preprocessing and subsequent data analyses were performed using the Statistical Parametric Mapping software (SPM12; Wellcome Department of Cognitive Neurology, London, UK; http://www.fil.ion.ucl.ac.uk). The preprocessing steps comprised: (1) slice-timing correction, (2) motion correction to correct for movement artifacts and related susceptibility artifacts through the re-alignment to the mean image; (3) coregistration of the anatomical and the functional images through a rigid-body registration of the mean functional image to the structural scan; (4) normalization of the functional acquisition to the Montreal Neurological Institute (MNI) standard space (Ashburner \& Friston, 2000) through the sequential application of a rigid body transformation and the nonlinear warp resultant of previous nonlinear registration of the structural scan to the MNI T1 template (5) regression of motion parameters, mean white matter (WM) and cerebrospinal fluid (CSF) signals; (6) smoothing with a 8-mm full-width halfmaximum Gaussian kernel to decrease spatial noise and (7) high pass temporal filtered (filter width of 128s).

Final images were visually inspected to guarantee that they had not any disproportionate head motion and that participants had no brain lesions. One participant was excluded due to head motion higher than $2 \mathrm{~mm}$ in translation and $1.5^{\circ}$ in rotation. In addition, two participants were excluded due anatomical abnormalities and two participants were excluded due to difficulties understanding the content of the videos. The fMRI paradigm analyses were performed using the general linear model (GLM) approach.

\section{Statistical analysis}

The design of the study is based on two main effects: the effect of condition (self or other) and the effect of the vignette emotional valence (positive, negative and neutral). First, to address the different neural responses to positive and negative videos in both self and other conditions, four different directional t-contrasts were computed. These contrasts included: self positive > self neutral, self negative $>$ self neutral, other positive $>$ other neutral and other negative $>$ neutral. Next, to assess each activation profile (e.g. activation in self condition when watching positive stimuli) one sample t-tests were computed. A family wise error (FWE) correction at voxel-level was adopted to correct for multiple corrections, with results being significant corrected for $\mathrm{FWE}<0.05$.

In addition, the above-mentioned contrast images were entered in repeated measures ANOVA with two within-subjects factors: condition (self and other) and valence (positive and negative). T-contrasts and an F-contrast were computed to assess factors main effects and the interaction effect. Results of the main effect of valence were corrected for multiple comparisons using FWE at voxel-level and results were considered significant for FWE $<0.05$. In addition, the results of the main effect of condition and interaction effect were thresholded at $p<0.005$ and cluster size $>15$ voxels. Small volume correction (SVC) with a threshold of $\mathrm{P} \leq 0.05$ was used to correct for multiple comparison. Regarding the SVC, the ROIs were selected from Bzdok et al. (2012), a meta-analysis that assesses both affective empathy and theory of mind regions based on $247 \mathrm{fMRI}$ experiments, including a total of 1790 participants. Finally, a combination of visual inspection and Anatomical Automatic Labeling atlas ( $A A L)$ was used for anatomical labelling (Tzourio-Mazoyer et al., 2002)

\section{Results}

\section{Self condition}

First, we looked at the brain's response to positive (versus neutral) and negative (versus neutral) stimuli, separately. In the self condition, the response to positive vignettes included brain activations in the bilateral middle and superior temporal gyrus (STG) - including the Helsch's gyri, TPJ, rolandic operculum, angular gyrus, postcentral, superior temporal poles (STP), and left posterior insula; bilateral precentral gyrus; bilateral SMA; left PCC and precuneus; bilateral ventral anterior thalamus; left medial dorsal thalamus; left IFG and left frontal inferior orbital extending to Al.

Still in the self condition, the brain's response to negative vignettes included the bilateral middle and STG - including the Helsch's gyri, left TPJ, rolandic operculum, STP and left posterior insula; bilateral precentral gyrus; left SMA; left PCC \& precuneus; bilateral inferior occipital gyrus; left fusiform, left ventral anterior thalamus and left IFG. Results are displayed in Figure 2 and Table 1. 


\section{Other condition}

Regarding the other condition, the neural response to positive vignettes (versus neutral) included the bilateral middle and STG - including the helsch's gyri, TPJ, angular gyrus, left SMG, posterior insula, STP and left rolandic operculum; left precentral gyrus; left PCC and precuneus; left IFG, bilateral middle/inferior occipital gyrus; left temporal inferior; bilateral ventral anterior thalamus; left medial dorsal thalamus; left midbrain and left frontal inferior orbital extending to Al.

Furthermore, the brain's response to negative vignettes (versus neutral) included activations in the bilateral middle and STG - including the helsch's gyri, TPJ, angular
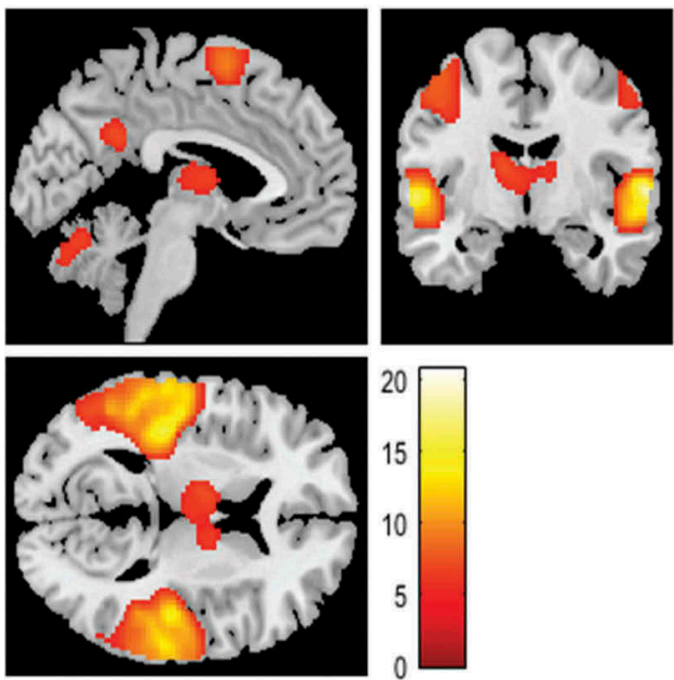

gyrus, middle and right STP, postcentral, SMG and left rolandic operculum; left precentral gyrus; left PCC \& precuneus, bilateral OI, left fusiform; left inferior temporal gyrus; left frontal inferior operulum and left frontal inferior orbital extending to anterior insula. Results are displayed in Figure 3 and Table 2.

\section{Comparing self/other condition}

Concerning the results of the ANOVA, the first factor (condition) showed significant differences in brain activity in both self $>$ other and other $>$ self direc-
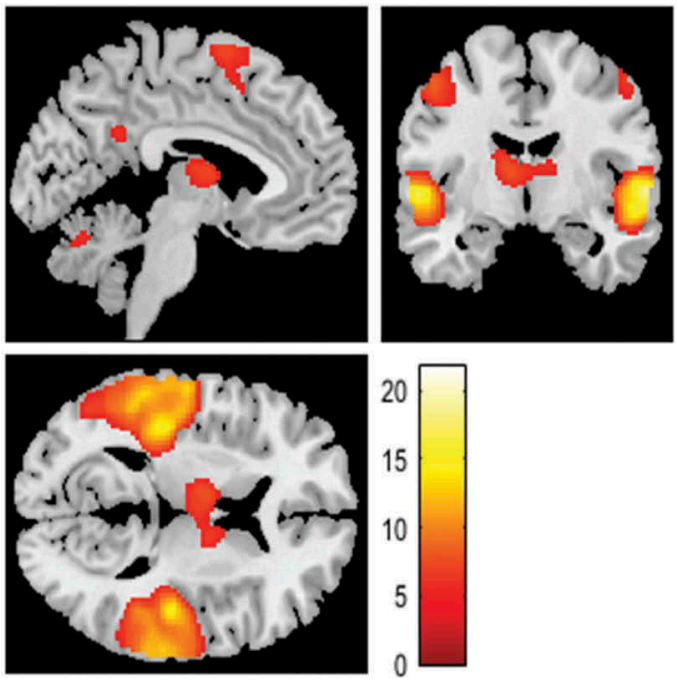

Figure 2. Activations identified in the self-condition when hearing to (a) positive (versus neutral) contents or (b) negative (versus neutral) contents expressed by their partner.

Table 1. Regional brain activity in the self condition for positive (> neutral) and negative (>neutral) stimuli.

\begin{tabular}{|c|c|c|c|c|c|c|}
\hline \multicolumn{2}{|r|}{ Region of interest } & $x$ & $y$ & z & Z & Cluster \\
\hline \multicolumn{7}{|c|}{ Positive > neutral } \\
\hline $\mathrm{L}$ & \multirow[t]{2}{*}{ Superior temporal gyrus } & -58 & -20 & 2 & $>8$ & 5509 \\
\hline $\mathrm{R}$ & & 56 & -24 & -2 & & 4391 \\
\hline $\mathrm{L}$ & \multirow[t]{2}{*}{ Precentral gyrus } & -40 & -4 & 48 & 7.22 & 728 \\
\hline $\mathrm{R}$ & & 52 & -2 & 52 & 6.65 & 288 \\
\hline $\mathrm{L}$ & \multirow[t]{2}{*}{ Supplementary motor area } & -2 & 4 & 66 & 6.57 & 512 \\
\hline $\mathrm{R}$ & & 4 & 12 & 64 & & \\
\hline $\mathrm{L}$ & Posterior cingulate \& precuneus & -10 & -50 & 30 & 6.14 & 301 \\
\hline $\mathrm{L}$ & Ventral anterior thalamus & 12 & -6 & 14 & 5.66 & 631 \\
\hline L & Medial dorsal thalamus & -2 & -12 & 8 & 5.19 & \\
\hline $\mathrm{R}$ & Ventral anterior thalamus & 10 & -4 & 12 & 5.18 & \\
\hline L & Frontal inferior orbital/Insula & -36 & 26 & -2 & 4.78 & 76 \\
\hline $\mathrm{L}$ & Inferior frontal gyrus & -50 & 18 & 18 & 4.77 & 50 \\
\hline \multicolumn{7}{|c|}{ Negative $>$ neutral } \\
\hline $\mathrm{L}$ & \multirow[t]{2}{*}{ Superior temporal gyrus } & -58 & -20 & 2 & $>8$ & 4764 \\
\hline $\mathrm{R}$ & & 56 & -24 & -2 & & 4090 \\
\hline $\mathrm{L}$ & \multirow[t]{2}{*}{ Precentral gyrus } & -40 & -4 & 48 & 7.09 & 725 \\
\hline $\mathrm{R}$ & & 52 & 0 & 52 & 6.91 & 230 \\
\hline $\mathrm{R}$ & \multirow[t]{2}{*}{ Inferior occipital gyrus } & 44 & -80 & -8 & 6.05 & 80 \\
\hline $\mathrm{L}$ & & -50 & -76 & -2 & 5.62 & \\
\hline $\mathrm{L}$ & Fusiform & -42 & -76 & -18 & 5.82 & 254 \\
\hline $\mathrm{L}$ & Ventral anterior thalamus & 10 & -6 & 12 & 5.89 & 471 \\
\hline $\mathrm{L}$ & Supplementary motor area & -2 & 4 & 66 & 5.81 & 389 \\
\hline $\mathrm{L}$ & Posterior Cingulate \& precuneus & -10 & -50 & 30 & 5.17 & 73 \\
\hline $\mathrm{L}$ & Inferior frontal gyrus & -48 & 18 & 20 & 4.66 & 39 \\
\hline
\end{tabular}

tions. The contrast self $>$ other evidenced significant clusters in the activity of the bilateral STC and insula. On the other hand, the contrast other $>$ self revealed several clusters, namely in the bilateral fusiform, right middle occipital gyrus, left supramarginal/postcentral (including insula), left inferior temporal gyrus, left precentral gyrus, left angular gyrus, and right temporal middle/TPJ. The summary of the results is displayed in Table 3 and Figure 4.

Regarding the second factor (valence), differences were only significant in the contrast positive $>$ negative video vignettes. Indeed, the brain's response to positive vignettes (versus negative) included brain activations in the left postcentral gyrus (including IPL) and right SMA. The summary of the results is displayed in Table 4.

Finally, an interaction effect between condition (self and other) and valence (positive and negative) was found in the left caudate with increased functional activity in the self condition for negative vignettes (see Table 3). 

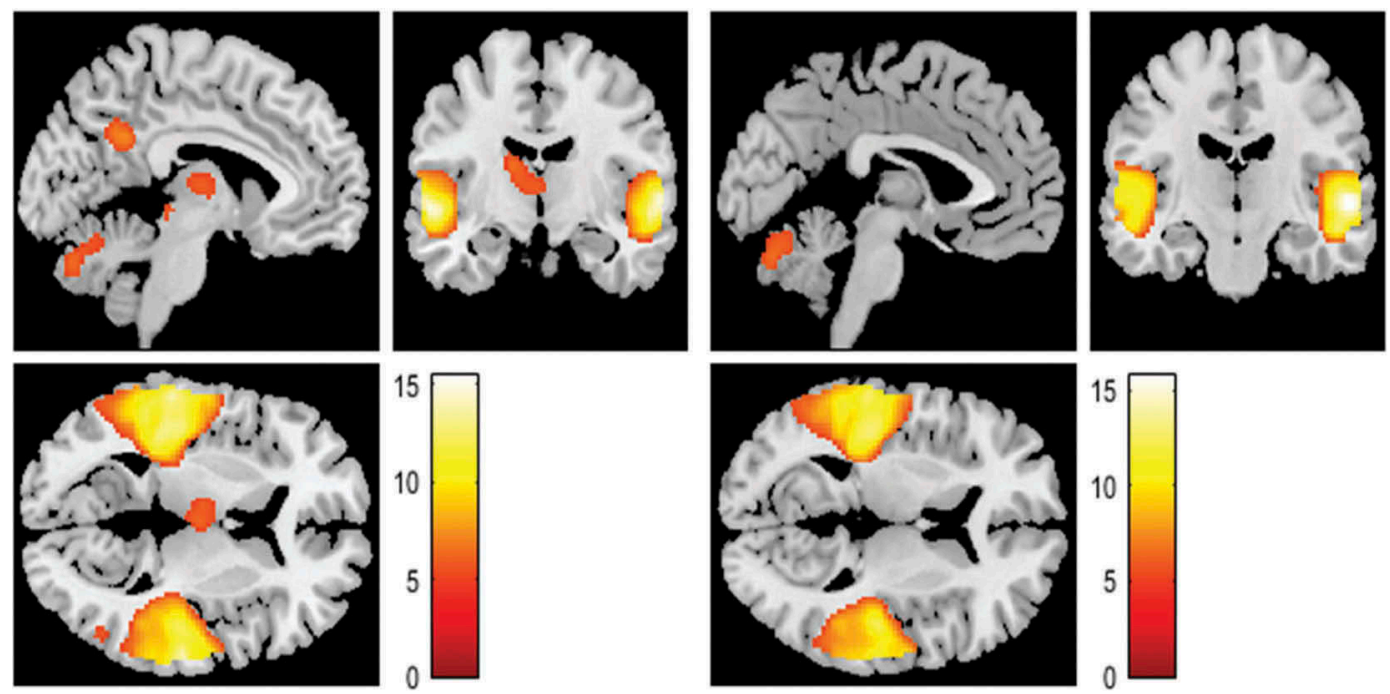

Figure 3. Activations identified in the other-condition when hearing to (a) positive (versus neutral) contents or (b) negative (versus neutral) contents expressed by their partner.

Table 2. Regional brain activity in the other condition for positive (vs neutral) and negative (vs neutral) stimuli.

\begin{tabular}{|c|c|c|c|c|c|c|}
\hline & Region of interest & $\mathrm{x}$ & $y$ & $\mathrm{z}$ & Z & Cluster \\
\hline \multicolumn{7}{|c|}{ Positive > Neutral } \\
\hline $\mathrm{L}$ & Superior temporal gyrus & -56 & -8 & -4 & $>8$ & 4951 \\
\hline $\mathrm{R}$ & & 58 & -14 & 0 & $>8$ & 3788 \\
\hline L & Precentral gyrus & -40 & -4 & 46 & 6.01 & 116 \\
\hline L & Posterior cingulate \& precuneus & -10 & -52 & 30 & 5.71 & 231 \\
\hline L & Inferior frontal gyrus & -48 & 14 & 22 & 5.50 & 109 \\
\hline $\mathrm{R}$ & Inferior occipital gyrus & 44 & -80 & -8 & 5.45 & 15 \\
\hline L & Inferior temporal gyrus & -46 & -50 & -18 & 5.30 & 325 \\
\hline L & Inferior occipital gyrus & -44 & -77 & -10 & 5.12 & \\
\hline L & Ventral anterior thalamus & -14 & -10 & 16 & 5.18 & 270 \\
\hline $\mathrm{L}$ & Medial dorsal thalamus & -2 & -12 & 8 & 4.99 & \\
\hline $\mathrm{L}$ & Frontal inferior orbital/Insula & -36 & 24 & -4 & 5.14 & 100 \\
\hline L & Midbrain & -4 & -28 & -4 & 5.00 & 16 \\
\hline $\mathrm{R}$ & Ventral anterior thalamus & 12 & -8 & 14 & 4.71 & 26 \\
\hline \multicolumn{7}{|c|}{ Negative $>$ neutral } \\
\hline L & Superior temporal gyrus & -56 & -8 & -4 & $>8$ & 4440 \\
\hline $\mathrm{R}$ & & 58 & -14 & 0 & $>8$ & 3618 \\
\hline L & Inferior occipital gyrus & -44 & -77 & -10 & 5.35 & 225 \\
\hline L & Fusiform & -42 & -71 & -18 & 5.12 & \\
\hline L & Precentral gyrus & -40 & -4 & 46 & 5.15 & 37 \\
\hline L & Inferior temporal gyrus & -42 & -46 & -14 & 5.11 & 32 \\
\hline $\mathrm{L}$ & Posterior cingulate \& precuneus & -10 & -52 & 30 & 4.99 & 67 \\
\hline L & Inferior frontal gyrus & -46 & 14 & 22 & 4.91 & 42 \\
\hline L & Frontal inferior orbital/Insula & -40 & -18 & -15 & 4.72 & 18 \\
\hline
\end{tabular}

\section{Discussion}

In this study, we looked at the neural basis of self/ other processing in the context of couple interaction during an fMRI task. In this task, each participant watched a set of video-vignettes of a previously recorded real interaction with his/her partner and was asked to focus either on what he/she was feeling (self condition) or on what his/her partner was feeling during the video (other condition). Consistent with our hypothesis, our results revealed a significant, although not complete, brain activity overlap between self and other conditions.
Table 3. Differences in brain activation between self and other conditions; Voxel Threshold: $p \leq .005$; Cluster Size Threshold: $k>15$.

\begin{tabular}{|c|c|c|c|c|c|c|}
\hline & \multirow[b]{2}{*}{ Region of interest } & \multicolumn{3}{|c|}{ MNI coordinates } & \multirow[b]{2}{*}{ Z } & \multirow[b]{2}{*}{ Cluster } \\
\hline & & $x$ & $Y$ & $\mathrm{Z}$ & & \\
\hline & Self $>$ Other & & & & & \\
\hline $\mathrm{L}$ & Superior temporal gyrus & -54 & -10 & 0 & 4.21 & 355 \\
\hline $\mathrm{L}$ & Insula & -44 & -14 & 2 & 4.08 & \\
\hline $\mathrm{R}$ & $\begin{array}{l}\text { Superior temporal gyrus/Insula } \\
\text { Other }>\text { Self }\end{array}$ & 48 & -8 & 4 & 3.69 & 82 \\
\hline L & Fusiform & -26 & -34 & -18 & 3.82 & 61 \\
\hline $\mathrm{R}$ & Middle occipital gyrus & 38 & -74 & 8 & 3.77 & 134 \\
\hline $\mathrm{R}$ & Fusiform & 28 & -36 & -16 & 3.55 & 101 \\
\hline L & Supramarginal gyrus/Postcentral & -54 & -20 & 18 & 3.23 & 142 \\
\hline L & Inferior temporal gyrus & -44 & -50 & -6 & 3.19 & 102 \\
\hline L & Precentral gyrus & -46 & 4 & 18 & 3.14 & 86 \\
\hline L & Angular gyrus & -36 & -62 & 30 & 3.12 & 53 \\
\hline $\mathrm{R}$ & $\begin{array}{l}\text { Temporal Middle* } \\
\text { Interaction effect }\end{array}$ & 50 & -48 & 18 & 2.87 & 32 \\
\hline L & Caudate & -18 & -18 & 24 & 3.71 & 425 \\
\hline
\end{tabular}

* Brain regions that survived small volume correction (SVC) at $p>0.05$ FWE with a $10 \mathrm{~mm}$ sphere using a priori independent coordinates from (Bzdok et al., 2012)

Both self and other conditions recruited brain regions associated with socio-affective processing (e.g., Al, anterior thalamus, MTG, IPL and IFG and PCC), as well as with sociocognitive processing (e.g., TPJ, precuneus and the STP (Bzdok et al., 2012; Schurz et al., 2014). The fact that both pathways of social processing were recruited, provides support to the idea of an interaction between affective and cognitive systems when understanding both ours and another's internal states (Lamm et al., 2010; Zaki et al., 2009).

Furthermore, the almost complete overlap between self and other neural activations brings support to the idea that understanding our own internal states and those of others requires similar psychological processes and associated brain mechanisms. This is consistent with 

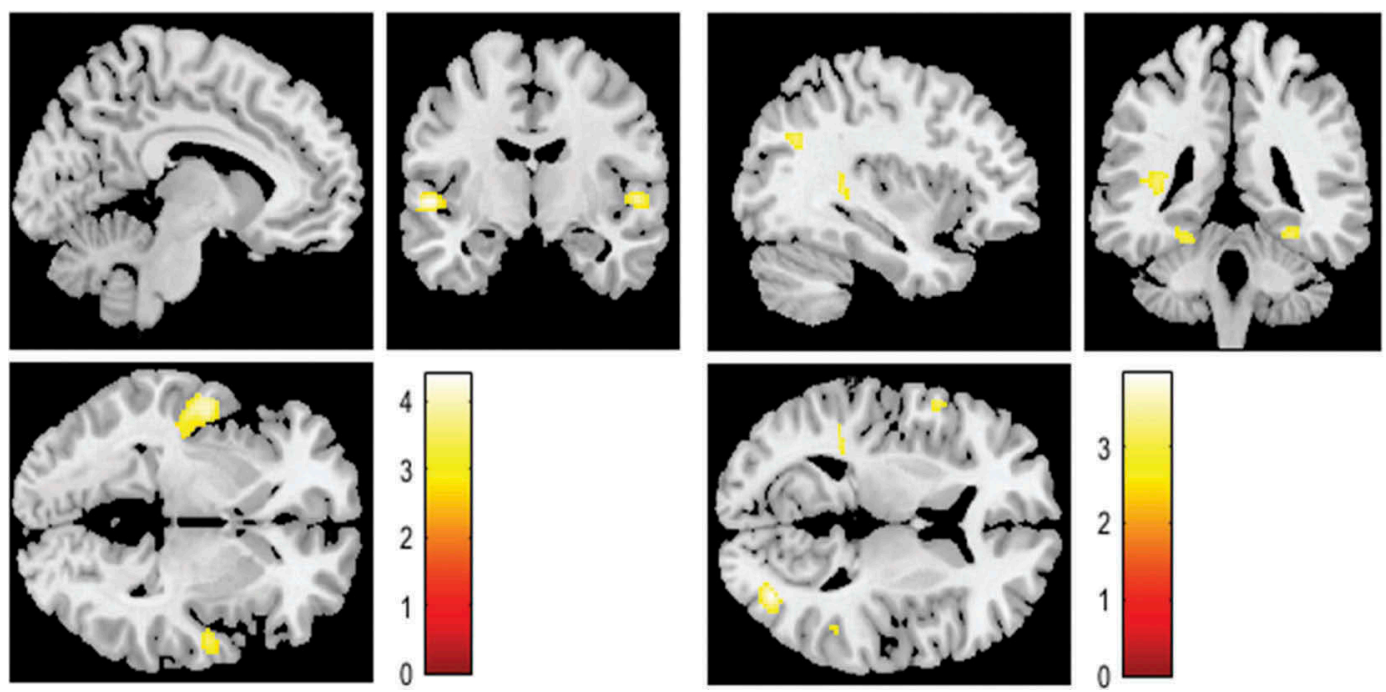

Figure 4. Activations identified when hearing to contents expressed by their partner for (a) self-condition higher than the other condition or (b) other-condition higher than the self-condition.

Table 4. Differences in brain activation for positive ( $>$ negative) contents expressed by their partner; Voxel Threshold: $p \leq .001$; FWE corrected; Cluster Size Threshold: k > 15.

\begin{tabular}{|c|c|c|c|c|c|c|}
\hline \multicolumn{5}{|c|}{ MNI coordinates } & \multirow[b]{2}{*}{ Z } & \multirow[b]{2}{*}{ Cluste } \\
\hline & Region of interest & $x$ & $y$ & $z$ & & \\
\hline \multirow[t]{2}{*}{$\mathrm{L}$} & Postcentral gyrus & -34 & -24 & 50 & 6.10 & 182 \\
\hline & Inferior parietal lobe & -46 & -24 & 38 & 5.24 & \\
\hline $\mathrm{R}$ & SMA & 6 & -14 & 48 & 4.82 & 45 \\
\hline
\end{tabular}

the "simulation"' theories of social cognition which argue that to understand the internal states of a close other we "simulate" them in ourselves (Adolphs, 2002; Gallese \& Goldman, 1998), engaging our own brain and body functions (Lamm et al., 2016; Rutgen, Seidel, Rie Ansky, \& Lamm, 2015). Indeed, the closer the other is to oneself, the more likely we are to base inferences about them on knowledge about oneself (Adolphs, 2002; Gallese \& Goldman, 1998).

Regarding the differences between conditions, while the self-condition was associated with higher activations in the bilateral STG region including the posterior insula, the other-condition was associated with higher activations in the bilateral fusiform, right middle occipital, left SMG, left temporal inferior, left precentral, left angular gyrus, and right temporal middle/TPJ. As expected, the self-condition engaged brain regions traditionally linked with interoceptive processing and affect sharing, such as the posterior insula (Chang et al., 2012; Craig, 2003; Singer et al., 2004). Previous work had reported increases in activation of the insula and the anterior cingulate cortex for self-related judgments when compared to other related judgments (e.g., Qin \& Northoff, 2011).

On the other hand, the other-condition recruited to a further extent brain areas involved in emotion recognition, such as the left inferior temporal gyrus, the bilateral fusiform and the left occipital middle (Adolphs, 2002; Hooker, Verosky, Germine, Knight, \& D'Esposito, 2008; Kawasaki et al., 2012) as well as in the representation of another's internal states/TOM, like the angular gyrus and the TPJ (Bzdok et al., 2012; Schurz et al., 2014). Importantly, the most significant region (the only one that survived to multiple comparison correction) for this contrast (other >self) was the right TPJ, a brain area thought to have a major role in differentiating self and other perspectives (Santiesteban, Banissy, Catmur, \& Bird, 2012; Steinbeis, 2016).

Indeed, the ability to distinguish and switch between self and other perspectives plays a key role in social cognitive processes (Santiesteban et al., 2012). Thus, the higher activation of the right TPJ in the other condition suggests that, in order to assume their partner's perspective, participants had to inhibit their own perspective (Steinbeis, 2016).

Furthermore, an interaction effect between emotional valence and condition was found in the caudate nucleus. Specifically, we found a higher activation in the left ventral caudate in the self-condition when participants were watching negative vignettes. Although the role of the caudate nucleus for self-referential processing remains unclear, previous evidence has shown that the left ventral caudate region is functionally connected to the posterior insula (Di Martino et al., 2008; Huang et al., 2017), a region known for interoceptive processing and affect sharing (Craig, 2003; Singer et al., 2004). In line with this result, recent evidence has shown that the functional connectivity between the posterior insula 
and the ventral caudate was significantly correlated with affective functions (Huang et al., 2017).

Finally, regarding the valence of the video-vignettes, in our study the neural response to positive vignettes was more evident than the response to negative vignettes. This increased activation was found in the right SMA and left postcentral gyrus including the IPL. These findings are not in line with previous data suggesting that humans have a remarkable ability to share the distress of others, but may react less to their joy (Perry, Hendler, \& ShamayTsoory, 2012). What our results seem to show is that when interacting with a close other such as an intimate partner, people may react more to positive emotions expressed by the other. In addition, our results showed that different brain areas were recruited for positive and negative stimuli. Specifically, positive contents were associated with higher activation in the bilateral thalamus, while negative stimuli were associated with higher activation in the inferior occipital gyrus and the fusiform gyrus - associated with self-referential processing of sad stimuli (Reniers, Völlm, Elliott, \& Corcoran, 2014). These results are in line with previous findings showing that depending on the valence of the stimuli, different brain areas are recruited (Phan, Wager, Taylor, \& Liberzon, 2002).

In summary, the present results show that there is an almost complete overlap between the neural systems that underlie processing our own internal states and those of a close other. In addition, the other condition recruited additional brain regions involved in the representation of another's internal states, TOM and selfother distinction. These findings provide support to simulationist accounts of social cognition which posits that our capacity to understand another's mind relies on our privileged access to our own mental states (Lamm et al., 2016; Preston \& de Waal, 2002; Rutgen et al., 2015). This may clarify why individuals with higher self-awareness are also more capable of understanding the other's emotions. This was recently shown by Böckler et al. (2017) that investigated the role of a training-induced understanding of oneself for the enhanced understanding of others. Results revealed that the degree to which participants improved their understanding of themselves predicted their improvements in high level ToM performance.

The results of this study have clear implications not only for couples functioning, but also for other human dyads such as mother-infant or helping professional relationships like physician-patient. They are also important for the understanding of clinical disorders in which the mechanisms of self and other processing are altered, such as depression or autism.

Future studies may build on the present results and move further to use analytic methods such as DCM that explicitly measure the influence that one neural system exerts over another. DCM analysis allows us to understand the flow of information within emotional and cognitive networks, as well as between networks. For example, the knowledge of the information flow between socioaffective and socio-cognitive networks, will clarify if these networks are hierarchically related, with the ability to abstract mental state attributions being dependent on the ability to simulate the other state.

\section{Disclosure statement}

No potential conflict of interest was reported by the authors.

\section{Funding}

This work was supported by BIAL Foundation [Grant number 87/12]. This study was conducted at Psychology Research Centre of University of Minho, and supported by the Portuguese Foundation for Science and Technology (FCT) and the Portuguese Ministry of Education and Science through national funds and co-financed by FEDER through COMPETE2020 under the PT2020 Partnership Agreement [POCI-01-0145-FEDER-007653]. Joana Coutinho was funded by a FCT postdoctoral Grant [UMINHO/BPD/10/2016]. Sofia Esménio was funded by a FCT doctoral Grant [PD/BD/ 105963/2014].

\section{ORCID}

Sofia Esménio (D) http://orcid.org/0000-0002-8155-1300

\section{References}

Adolphs, R. (2002). Neural systems for recognizing emotion. Current Opinion in Neurobiology, 1-9. Figure 1. doi:10.1016/ S0959-4388(02)00301-X

Ashburner, J., \& Friston, K. J. (2000). Voxel-based morphometry-The methods. Neurolmage, 11, 805-821.

Batson, C. D., Lishner, D. A., Cook, J., \& Sawyer, S. (2005). Similarity and nurturance: Two possible sources of empathy for strangers. Basic and Applied Social Psychology, 27(1), 15-25.

Beach, S. R. H., Fincham, F. D., \& Katz, J. (1998). Marital therapy in the treatment of depression: Toward a third generation of therapy and research. Clinical Psychology Review, doi:10.1016/S0272-7358(98)00023-3

Böckler, A., Herrmann, L., Trautwein, F.-M., Holmes, T., \& Singer, T. (2017). Know thy selves: Learning to understand oneself increases the ability to understand others. Journal of Cognitive Enhancement, 1(2), 197-209.

Bzdok, D., Schilbach, L., Vogeley, K., Schneider, K., Laird, A. R., Langner, R., \& Eickhoff, S. B. (2012). Parsing the neural correlates of moral cognition: ALE meta-analysis on morality, theory of mind, and empathy. Brain Structure and Function, 217(4), 783-796. 
Carrington, S. J., \& Bailey, A. J. (2009). Are there theory of mind regions in the brain? A review of the neuroimaging literature. Human Brain Mapping, 30(8), 2313-2335.

Carvalho, S., Leite, J., Galdo-Álvarez, S., \& Gonçalves, Ó. F. (2012). The emotional movie database (EMDB): A self-report and psychophysiological study. Applied Psychophysiology Biofeedback, 37(4), 279-294.

Chang, L. J., Yarkoni, T., Khaw, M. W., \& Sanfey, A. G. (2012). Decoding the role of the insula in human cognition: Functional parcellation and large-scale reverse inference. Cerebral Cortex, 23(3), 739-749.

Cialdini, R., Brown, S., Lewis, B., Luce, C., \& Neuberg, S. (1997). Reinterpreting the empathy-Altruism relationship: When one into one equals oneness. Journal of Personality and Social Psychology, 73(3), 481-494. Retrieved from: http:// pds4.egloos.com/pds/200702/06/77/c_documents_and_set tings_owner_local_settings_application_data_mozilla_fire fox_profiles_mv4mjwqz.pdf

Coutinho, J., Oliveira-Silva, P., Fernandes, E., Gonçalves, O. F., Correia, D., Perrone Mc-Govern, K., \& Tschacher, W. (2018). Psychophysiological synchrony during verbal interaction in romantic relationships. Family Process. doi:10.1111/ famp.12371

Coutinho, J., Oliveira-Silva, P., Mesquita, A. R., Barbosa, M., Perrone-Mcgovern, K. M., \& Gonçalves, O. F. (2017). Psychophysiological reactivity in couples during a marital interaction task. Applied psychophysiology and biofeedback, 42, 335-346.

Coyne, J. C., Rohrbaugh, M. J., Shoham, V., Sonnega, J. S., Nicklas, J. M., \& Cranford, J. A. (2001). Prognostic importance of marital quality for survival of congestive heart failure. The American Journal of Cardiology, 88. Retrieved from http://userpage.fu-berlin.de/ health/materials/ coyne1.pdf

Craig, A. D. (2003). Interoception: The sense of the physiological condition of the body. Current Opinion in Neurobiology, 13(4), 500-505.

Cummings, E. M., \& Davies, P. T. (2002). Effects of marital conflict on children: Recent advances and emerging themes in process-oriented research. Journal of Child Psychology and Psychiatry, 43(1), 31-63. Retrieved from: https://www.research gate.net/profile/Patrick_Davies/publication/11513668_ Effects_of_marital_conflict_on_children_Recent_advances_ and_emerging_themes_in_process-oriented_research/links/ 59e73b9c0f7e9b13acaca53a/Effects-of-marital-conflict-onchildren-Recent

de Vignemont, F., \& Singer, T. (2006). The empathic brain: How, when and why? Trends in Cognitive Sciences, 10(10), 435-441.

Decety, J., \& Lamm, C. (2006). Human empathy through the lens of social neuroscience. The Scientific World Journal, 6, 1146-1163.

Di Martino, A., Scheres, A., Margulies, D. S., Kelly, A. M. C., Uddin, L. Q., Shehzad, Z., ... Milham, M. P. (2008). Functional connectivity of human striatum: A resting state fMRI study. Cerebral Cortex, 18(12), 2735-2747.

Dimaggio, G., Lysaker, P. H., Carcione, A., Nicolò, G., \& Semerari, A. (2008). Know yourself and you shall know the other... to a certain extent: Multiple paths of influence of self-reflection on mindreading. Consciousness and Cognition, 17(3), 778-789.
Fan, Y., Duncan, N. W., de Greck, M., \& Northoff, G. (2011). Is there a core neural network in empathy? An fMRI based quantitative meta-analysis. Neuroscience and Biobehavioral Reviews, 35(3), 903-911.

Frith, C., \& Frith, U. (2005). Theory of mind. Current Biology, 15 (17), R644-R645.

Gallese, V., \& Goldman, A. (1998). Mirror neurons and the mind-reading. Trens in Cognitive Sciences, 2(12), 493-501.

Greene, S., \& Griffin, W. (1998). Symptom study in context: Effects of marital quality on signs of Parkinson's disease during patient-spouse interaction. Psychiatry, 61(1), 35-45.

Gottman, J. M., \& Levenson, R. W. (1988). The social psychophysiology of marriage. In P. Noller \& M. A. Fitzpatrick (Eds.), Monographs in social psychology of language, No. 1. Perspectives on marital interaction (pp. 182-200). Clevedon, England: Multilingual Matters.

Hooker, C. I., Verosky, S. C., Germine, L. T., Knight, R. T., \& D'Esposito, M. (2008). Mentalizing about emotion and its relationship to empathy. Social Cognitive and Affective Neuroscience, 3(3), 204-217.

Huang, H., Nguyen, P. T., Schwab, N. A., Tanner, J. J., Price, C. C., \& Ding, M. (2017). Mapping dorsal and ventral caudate in older adults: Method and validation. Frontiers in Aging Neuroscience, 9(APR). doi:10.3389/fnagi.2017.00091

Kanske, P., Böckler, A., Trautwein, F.-M., \& Singer, T. (2015). Dissecting the social brain: Introducing the EmpaToM to reveal distinct neural networks and brain-behavior relations for empathy and theory of mind. Neurolmage, 122, 6-19.

Kawasaki, H., Naotsugu, T., Kovach, C. K., Nourski, K. V., Oya, H., Howard, M. A., \& Adolphs, R. (2012). Processing of facial emotion in the human fusiform gyrus. Journal of Cognitive Neuroscience, 24(6), 1358-1370.

Khalsa, S. S., Adolphs, R., Cameron, O. G., Critchley, H. D., Davenport, P. W., Feinstein, J. S., ... Paulus, M. P. (2017). Review interoception and mental health: A roadmap. doi:10.1016/jbpsc.2017.12.004

Lamm, C., Bukowski, H., \& Silani, G. (2016). From shared to distinct self-Other representations in empathy: Evidence from neurotypical function and socio-cognitive disorders. Philosophical Transactions of the Royal Society B: Biological Sciences, 371(1686), 20150083.

Lamm, C., Decety, J., \& Singer, T. (2010). Meta-analytic evidence for common and distinct neural networks associated with directly experienced pain and empathy for pain. Neurolmage, 54(3), 2492-2502.

Lombardo, M. V., Chakrabarti, B., Bullmore, E. T., Wheelwright, S. J., Sadek, S. A., Suckling, J., ... Consortium, M. A. (2010). Shared neural circuits for mentalizing about the self and others. Journal of Cognitive Neuroscience, 22(7), 1623-1635.

Mayne, T. J., O'Leary, A., McCrady, B., Contrada, R., \& Labouvie, E. (1997). The differential effects of acute marital distress on emotional, physiological and immune functions in maritally distressed men and women. Psychology \& Health, 12(2), 277-288. http://dx.doi.org/10.1080/08870449708407405

Mitchell, J. P., Macrae, C. N., \& Banaji, M. R. (2006). Dissociable medial prefrontal contributions to judgments of similar and dissimilar others. Neuron, 50(4), 655-663.

Péloquin, K., \& Lafontaine, M.-F. (2010). Measuring empathy in couples: Validity and reliability of the interpersonal reactivity index for couples. Journal of Personality Assessment, 92 (June 2012), 146-157. 
Perry, D., Hendler, T., \& Shamay-Tsoory, S. G. (2012). Can we share the joy of others? Empathic neural responses to distress vs joy. Social Cognitive and Affective Neuroscience, 7(8), 909-916.

Phan, K. L., Wager, T., Taylor, S. F., \& Liberzon, I. (2002). Functional neuroanatomy of emotion: A meta-analysis of emotion activation studies in pet and fMRI. Neuroimage, 16 (2), 331-348.

Preston, S. D., \& de Waal, F. B. M. (2002). Empathy: Its ultimate and proximate bases. Behavioral and Brain Sciences, 25(01), $1-71$.

Qin, P., \& Northoff, G. (2011). How is our self related to midline regions and the default-mode network?. Neurolmage, 57(3), $1221-1233$.

Reniers, R L. E. P., Völlm, B. A., Elliot, R., \& Corcoran, R. (2014). Empathy, TOM and self-other differentiation: An fMRI study of internal states. Social Neuroscience, 9,(1). 10.1080/ 17470919.2013.861360

Robles, T. F., \& Kiecolt-Glaser, J. K. (2003). The physiology of marriage: Pathways to health. Physiology \& Behavior, 79, 409-416.

Rutgen, M., Seidel, E.-M., Rie Ansky, I., \& Lamm, C. (2015). Reduction of empathy for pain by Placebo Analgesia suggests functional equivalence of empathy and first-hand emotion experience. Journal of Neuroscience, 35(23), 8938-8947.

Santiesteban, I., Banissy, M. J., Catmur, C., \& Bird, G. (2012). Enhancing social ability by stimulating right temporoparietal junction. Current Biology, 22(23), 2274-2277.

Santiesteban, I., White, S., Cook, J., Gilbert, S. J., Heyes, C., \& Bird, G. (2012). Training social cognition: From imitation to theory of mind. Cognition, 122(2), 228-235.

Schurz, M., Radua, J., Aichhorn, M., Richlan, F., \& Perner, J. (2014). Fractionating theory of mind: A meta-analysis of functional brain imaging studies. Neuroscience and Biobehavioral Reviews, 42, 9-34.

Singer, T., \& Lamm, C. (2009). The social neuroscience of empathy. Annals of the New York Academy of Sciences, 1156, 81-96.

Singer, T., Seymour, B., O'Doherty, J., Dolan, R. J., Kaube, H., \& Frith, C. D. (2004). Empathy for pain involves the affective but not sensory components of pain. Science (New York, N. $Y, 303(5661), 1157-1162$.

Singer, T., Seymour, B., O'Doherty, J. P., Stephan, K. E., Dolan, R. J., \& Frith, C. D. (2006). Empathic neural responses are modulated by the perceived fairness of others. Nature, 439(7075), 466-469.

Steinbeis, N. (2016). The role of self - Other distinction in understanding others ' mental and emotional states: Neurocognitive mechanisms in children and adults. Philosophical Transactions of the Royal Society B: Biological Sciences, 371 (1686), 20150074.

Tzourio-Mazoyer, N., Landeau, B., Papathanassiou, D., Crivello, F., Etard, O., Delcroix, N., ... Joliot, M. (2002). Automated anatomical labeling of activations in SPM using a macroscopic anatomical parcellation of the MNI MRI single-subject brain. Neurolmage, 15(1), 273-289.

Waldinger, R. J., Hauser, S. T., Schulz, M. S., Allen, J. P., \& Crowell, J. A. (2004). Reading others emotions: The role of intuitive judgments in predicting marital satisfaction, quality, and stability. Journal of Family Psychology?: JFP?: Journal of the Division of Family Psychology of the American Psychological Association (Division 43), 18(1), 58-71.

Zaki, J., Weber, J., Bolger, N., \& Ochsner, K. (2009). The neural bases of empathic accuracy. Proceedings of the National Academy of Sciences, 106(27), 11382-11387. 